\title{
Single-cell RNA Sequencing Reveals Transcriptional Signatures and Cell-cell Crosstalk in Patients With Hypertensive Nephropathy
}

Rong Tang

Xiangya Hospital Central South University

Wei Lin

Xiangya Hospital Central South University

Chanjuan Shen

Central South University Xiangya Medical College: Central South University Xiangya School of Medicine

Ting Meng

Xiangya Hospital Central South University

Joshua D Ooi

Monash University

Peter J Eggenhuizen

Monash University

Peng Jin

Xiangya Hospital Central South University

Xiang Ding

Xiangya Hospital Central South University

Jinbiao Chen

Xiangya Hospital Central South University

Wannian Nie

Xiangya Hospital Central South University

\section{Xiang Ao}

Xiangya Hospital Central South University

\section{Weisheng Peng}

Xiangya Hospital Central South University

Qiaoling Zhou

Xiangya Hospital Central South University

\section{Ping Xiao}

Xiangya Hospital Central South University

Yong Zhong ( $\square$ zhongyong121@163.com )

Xiangya Hospital Central South University https://orcid.org/0000-0003-2520-0087

Xiangcheng Xiao 


\section{Research Article}

Keywords: Hypertensive nephropathy, Single-cell RNA sequencing, Differentially expressed genes, Cell-cell crosstalk

Posted Date: June 21st, 2021

DOI: https://doi.org/10.21203/rs.3.rs-599634/v1

License: (1) This work is licensed under a Creative Commons Attribution 4.0 International License. Read Full License 


\title{
Single-cell RNA sequencing reveals transcriptional signatures and cell-cell crosstalk in patients with hypertensive nephropathy
}

Rong Tang ${ }^{1 *}$, Wei $\mathrm{Lin}^{2 *}$, Chanjuan Shen ${ }^{3}$, Ting Meng ${ }^{1}$, Joshua D. Ooi ${ }^{1,4}$, Peter J. Eggenhuizen ${ }^{4}$, Peng Jin ${ }^{5}$, Xiang Ding ${ }^{5}$, Jinbiao $\mathrm{Chen}^{6}$, Wannian $\mathrm{Nie}^{1}$, Xiang $\mathrm{Ao}^{1}$, Weisheng Peng ${ }^{1}$, Qiaoling Zhou ${ }^{1}$, Ping Xiao ${ }^{1}$, Yong Zhong ${ }^{1 \#}$, Xiangcheng Xiao ${ }^{1 \#}$

"These authors have contributed equally to this work and share first authorship.

Running head: ScRNA-seq of hypertensive nephropathy

${ }^{\#}$ Corresponding author:

Yong Zhong, 87 Xiangya Road, Changsha, Hunan 410008, P.R. China, E-mail: zhongyong121@163.com, Tel.: +86-0731-84327431;

Xiangcheng Xiao, 87 Xiangya Road, Changsha, Hunan 410008, P.R. China, E-mail: 1376785378@qq.com, Tel.: +86-0731-84327431.

\begin{abstract}
Background: Hypertensive nephropathy (HTN) is one of the leading causes of end-stage renal disease, yet the precise mechanisms and cell-specific gene expression changes are still unknown. This study used single-cell RNA sequencing (scRNA-seq) to explore novel molecular mechanisms and gene targets for HTN for the first time.

Methods: The gene expression profiles of renal biopsy samples obtained from HTN patients and healthy living donor controls were determined by scRNA-seq technology. Distinct cell clusters, differential gene expression, cell-cell interaction and potential signaling pathways involved in HTN were determined.

Results: 18 distinct cell clusters were identified in kidney from HTN and control subjects. Endothelial cells overexpressed $L R G 1$, a pleiotropic factor linked to apoptosis and inflammation, providing a potential novel molecular target. HTN endothelium also overexpressed genes linked to cellular adhesion, extracellular matrix accumulation and inflammation. In HTN patients, mesangial cells highly expressed proliferation related signatures (MGST1, TMSB10, EPS8 and IER2) not detected in renal diseases before. The upregulated genes in tubules of HTN were mainly
\end{abstract}


participating in inflammatory signatures including IFN- $\gamma$ signature, IL-17 signaling and TLR signaling. Specific gene expression of kidney-resident $\mathrm{CD} 8^{+} \mathrm{T}$ cells exhibited a proinflammatory, chemotactic and cytotoxic phenotype. Furthermore, receptor-ligand interaction analysis indicated cell-cell crosstalk in kidney contributes to recruitment and infiltration of inflammatory cells into kidneys, and fibrotic process in hypertensive renal injury.

Conclusions: In summary, our data identifies a distinct cell-specific gene expression profile, pathogenic signaling pathways and potential cell-cell communications in the pathogenesis of HTN. These findings will provide a promising novel landscape for mechanisms and treatment of HTN.

Keywords: Hypertensive nephropathy, Single-cell RNA sequencing, Differentially expressed genes, Cell-cell crosstalk

\section{Background}

Hypertension is a major risk factor for ischemic heart disease, heart failure, stroke and chronic kidney diseases (CKD) [1]. It is estimated that around one third of people suffer from hypertension worldwide, and hypertension also serves as an important global public-health challenge [2,3]. Hypertensive nephropathy (HTN) is highly prevalent and is regarded as one of the leading causes of end-stage renal disease (ESRD), leading to significant morbidity and mortality [4,5]. The prevalence of hypertension associated ESRD continues to rise annually, which brings a heavy burden on family, society and healthcare financing $[6,7]$.

Multiple molecular mechanisms are involved in the promotion of hypertensive renal damage, such as genetic factors, renin-angiotensin-aldosterone system (RAAS) activation, endothelial dysfunction, impaired capillary blood flow, inflammation and oxidative stress [8]. Until now, the exact pathogenesis underlying HTN is complex and still not fully elucidated. At present, strict control of blood pressure including utilization of angiotensin-converting enzyme inhibitors (ACEI) or angiotensin II receptor blockers (ARB) has been considered as an effective measure, yet well controlled blood pressure slows but can't fully prevent the progression of renal damage caused by hypertension [9]. Although great efforts have been made in 
combating and preventing HTN, there is still a shortage of effective treatments. Thus, identification of novel mechanisms underlying HTN and improvement of early diagnosis and treatment strategies are key issues in HTN.

It is challenging to elucidate the mechanisms responsible for the initiation and development of HTN due to the complexity of kidney structure and function. Single-cell RNA sequencing (scRNA-seq) has emerged as a powerful scientific method which grew as an alternative to traditional bulk RNA sequencing (RNA-seq) technology [10]. Bulk RNA-seq method can only profile the average expression of individual genes across a diverse group of cells in the sample, and is unable to identify transcriptional features of individual cells [11]. Unlike tissue-level bulk RNA-seq, scRNA-seq technology defines gene transcription and heterogeneity at the single-cell level, and shows great potential to identify novel cell types and subpopulations. Moreover, scRNA-seq technology enables the analysis of transcriptomes and signaling pathways simultaneously in various cell clusters extracted from tissues $[12,13]$. This new methodology has already been used in the field of kidney diseases, including lupus nephritis, diabetic nephropathy, acute kidney injury and IgA nephropathy [14-17].

In the present study, for the first time we performed scRNA-seq to analyze gene expression at the single cell level in human renal biopsies of patients with hypertensive nephropathy and pre-transplant healthy living controls. Our findings identified different cell types and detected potential novel cellular mechanisms and molecules involved in progression of HTN. We also revealed valuable data about crucial intercellular and cell-type-specific signaling pathways driving disease pathogenesis. These investigations will contribute to developing potential novel therapeutic targets for the treatment of hypertensive renal damage in the future.

\section{Materials and methods}

\section{Ethics approval and consent}

Renal tissue samples were obtained as portion of the Kidney Precision Medicine Study which was approved by the Medical Ethics Committee of Xiangya Hospital of Central South University for Human Studies (No. 201711836). All subjects needed written informed consent, and all experiments were conducted according to the research protocol. 


\section{Clinical sample}

Kidney samples from two newly diagnosed patients with HTN were collected from the department of nephrology in Xiangya Hospital of Central South University. HTN was diagnosed histologically by renal pathological biopsy, and the predominant histological findings were myointimal hyperplasia of arterioles, arteriolar hyalinosis, wrinkling of basement membrane, and focal renal tubulointerstitial inflammation and fibrosis. Kidney tissues from renal core 18-gauge needle biopsies essential for clinical diagnosis were collected from consented subjects with HTN. One control kidney specimen was obtained by needle biopsy from living donor kidneys after removal from the donors, and prior to implantation into the recipients. The estimated glomerular filtration rate (eGFR) was caculated by CKD-EPI creatinine formula. ScRNA-seq dataset of another healthy adult kidney sample has already been deposited at Gene Expression Omnibus (GEO) under accession number GSE171314. The raw sequencing data Only a small amount (2-3 $\mathrm{mg}$ ) of renal biopsy was acquired for the scRNA-seq procedure. Kidney tissues were washed with sterile PBS after collection.

\section{Kidney tissue processing and single-cell dissociation}

The fresh renal biopsy was immediately put into the GEXSCOPE Tissue Preservation Solution (Singleron Biotechnologies) at $2-8^{\circ} \mathrm{C}[18]$. The biopsy sample was washed three times with Hanks' Balanced Salt Solution (HBSS), and subsequently cut into small pieces (1-2 mm). The sample was then digested in 2ml GEXSCOPE Tissue Dissociation Solution (Singleron Biotechnologies) in a $15 \mathrm{ml}$ centrifuge tube by continuous agitation for $15 \mathrm{~min}$ at $37^{\circ} \mathrm{C}$. Subsequently, the sample was filtered using a $40 \mu \mathrm{m}$ sterile cell strainer (Corning). The solution was centrifuged at $1000 \mathrm{rpm}$ for 5 min at $4^{\circ} \mathrm{C}$, cell pellets were resuspended in $1 \mathrm{ml}$ PBS (HyClone). The cell suspension was treated with $2 \mathrm{ml}$ GEXSCOPE Red Blood Cell Lysis Buffer (Singleron Biotechnologies) for $10 \mathrm{~min}$ at $25^{\circ} \mathrm{C}$ to remove red blood cells. Cell pellet was resuspended in PBS after centrifugation at $1000 \mathrm{rpm}$ for $5 \mathrm{~min}$. Cells were then enumerated through a TC20 automated cell counter (Bio-Rad). Live cells were identified by trypan blue staining (Gibco). If cell viability was more than $80 \%$, the subsequent processing of sample was conducted. 


\section{Library preparation and scRNA-seq data preprocessing}

The single cell suspension was adjusted in PBS to a density of $1 \times 10^{5}$ cells $/ \mathrm{mL}$. Then the single cell suspension was loaded onto a microfluidic chip. The preparation of scRNA-seq library was performed following the manufacturer's protocol (Singleron GEXSCOPE Single Cell RNA-seq Library Kit, Singleron Biotechnologies) [18]. Illumina HiSeq X10 instrument and 150 bp paired-end reads were used to sequence the captured scRNA-seq libraries. The scRNA sequencing was deposited in Gene Expression Omnibusdatabase at NCBI.

\section{Quality control and marker gene analysis in different cell clusters}

Cells with less than 200 or more than 5000 expressed genes were discarded in this study. We removed cells that had higher than 30,000 UMIs and mitochondria content more than $50 \%$.After identification of cell clusters, the marker genes of each cell group in the kidney were identified relative to other cell clusters by the "Wilcox" (Likelihood-ratio test) using the FindAllMarkers function of Seurat. Avg.exp represented the average expression of the gene in each cell type. The marker genes were selected based on expression in more than $10 \%$ of cells in one cell group, and the average log (Fold Change) being greater than 0.25 . The heatmap was generated by identification of the top20 marker genes from each cell group

\section{Identification of differentially expressed genes between groups}

We identified differentially expressed genes (DEGs) in each kidney cell population by comparing the transcriptional profiles of HTN patients with controls. Using the FindAllMarkers function in Seurat, the DEGs of each cell population between two groups were determined by the "wilcox" (Likelihood-ratio test). A gene with an absolute average log (Fold Change) value exceeding 0.25 and with a $P$ value less than 0.05 was identified as a differentially expressed gene.

\section{Enrichment and receptor-ligand analysis}

Gene Ontology (GO) and Kyoto Encyclopedia of Genes and Genomes (KEGG) enrichment analysis were carried out using the "clusterProfiler" $\mathrm{R}$ package to clarify the functional effects of DEGs. Receptor-ligand interaction network between distinct cell clusters was analyzed by CellphoneDB. 


\section{Results}

A total of 4 kidney samples from patients with $\operatorname{HTN}(n=2)$, and healthy living donor controls $(n=2)$ were processed. In our study, one HTN patient was a 45-year-old man with a history of hypertension for 6 years. Another HTN patient was a 53-year-old man with a history of hypertension for 8 years. Both of these two HTN patients had proteinuria. HTN patients had no concurrent diseases including diabetes or primary renal disease. Other secondary kidney diseases were excluded either. One living donor was a 41-year-old male, another living donor was a 48-year-old male. Both of the living donors have no history of diseases such as hypertension and diabetes, and have not been prescribed any medications before.

\section{Cell lineage identification in human renal cortical tissue}

We obtained kidney specimens freshly from HTN and healthy subjects, then separated and digested them into a single cell suspension. Subsequently, scRNA-seq was conducted. Cell yield totaled 19,964 cells (HTN subjects had 8661 cells in total) from four specimens, and cell viability was between $84.50 \%$ and $96.55 \%$ (Supplemental Table. 1). After data processing and quality control were carried out, transcriptome profiles were analyzed. We used unsupervised clustering to visualize renal cell populations, and cell populations were identified based on anchor gene expression of each cluster and from the literature. UMAP plots of 18 cell clusters from the kidney are shown in Fig. 1a, including mesangial cells, endothelial cells, podocytes, proximal and distal tubular cells, loop of Henle cells, principal and intercalated cells from collecting ducts, pericytes, macrophages, dendritic cells, monocytes and $\mathrm{T}$ cells. The distribution of distinct cell clusters from individual subjects with HTN and the control subjects is represented as a UMAP plot (Fig. 1b). The percentages of different cell populations in each subject were defined respectively (Fig. 1c). A heatmap of the top 20 most differentially expressed marker genes for each cell cluster was used to determine mutually exclusive genomes and to distinguish cell lineage (Fig. 1d and Supplemental dataset. 1). Violin plots of a representative selected marker for each cell population are illustrated in Fig. 1e. A complete list of cell-type-specific DEGs is provided in Supplemental dataset. 1-3.

Cell clustering was identified by mutually exclusive expression of cell lineage-specific marker genes (Table. 1). For example, high expression of FN1, FHL2 
and MYL9 identified this cell cluster as mesangial cells, while the expression of NPHS2, PODXL and PTPRO characterized podocytes. Endothelial cells uniquely expressed PECAM1, KDR and CLDN5, whereas fibroblasts expressed distinct genes (COL1A1, DCN and $L U M$ ). For renal tubular epithelial cells, proximal tubule cells distinctly expressed $A L D O B, L R P 2, S L C 13 A 1$ and $C U B N$, while distal tubule cells were identified by expression of CALB1 and SLC12A3. Loop of Henle cells expressed UMOD, SLC12A1 and CLDN16. Marker genes AQP2, AQP3 and GATA3 identified principle cells, while SLC4A1, ATP6VOD2, FOXI1 and DMRT2 defined intercalated cells. Leukocytes expressed distinct T cell, B cell, macrophage and plasma cell genes (CD3G, MS4A1, CD68 and IGHG1, respectively).

\section{Transcriptomics of endothelium in kidney involved in the pathogenesis of HTN}

Endothelial dysfunction is a key factor contributing to the development of hypertension and renal damage. In HTN renal tissues, endothelial cells highly expressed $L R G 1$, a pleiotropic molecular, which has a pathogenic effect in multiple human diseases [19]. LRG1 participated in pathological and biological processes including cell adhesion, protein-protein interactions and signal transduction. LRG1 was also shown to facilitate apoptosis and autophagy through the TGF- $\beta 1 /$ ALK1-mediated signaling pathway [20]. We proposed that endotheliumderived LRG1 might be a novel molecular target in the pathophysiology of HTN.

In addition to $L R G 1$, the endothelium also overexpressed cellular adhesion genes including SELP, CEACAM1 and PECAM1, probably mediating the aggregation and adhesion of inflammatory cells to the injured site. $A C K R 1$, enriched at the endothelial junction, modulates chemokine levels and helps leukocyte recruitment [21]. We found ACKRl gene expression was also increased in HTN endothelial cells. CCL23 and CCL2, chemokines participating in leukocyte trafficking and the inflammatory response, were overexpressed in the endothelium. $V W A 1$, which played a role in collagen synthesis [22]. was upregulated in HTN endothelial cells and not reported in renal pathology before. Interestingly, For HTN patients, the endothelium highly expressed the hyaluronan (HA)-binding receptor $L Y V E-1$, which facilitated collagen expression and fibrosis through engagement with the HA and extracellular matrix (ECM) [23]. Functional analysis indicated upregulated DEGs were enriched in tumor necrosis factor (TNF) signaling, cellular response to TNF or interferon-gamma (IFN- $\gamma$ ), focal adhesion and cell-substrate adhesion (Fig. 2b and 2c, Supplemental 
dataset. 5 and 6). These data reveal endothelial cells overexpressed genes which are linked to cellular adhesion, inflammation and ECM accumulation, indicating that the endothelium plays an initial and promoting role in the pathogenesis of HTN.

\section{Cell-cluster-specific gene expression revealed molecular signatures of the glomerulus in HTN}

Mesangial cells, pericytes and podocytes in glomerulus were identified based on cell-cluster-specifically expressed genes. We also analyzed DEGs in the glomerulus through comparing the transcripts between HTN and control subjects (Fig. 2 and Supplemental dataset. 2).

HTN mesangial cells were found to highly express proliferation related signatures. Compared to the control samples, expression of several genes (MGST1, TMSB10, EPS8 and IER2) was increased in mesangial cells from HTN patients and has not been investigated previously in renal diseases. MGST1, a membrane bound glutathione transferase, has been shown to play crucial roles in cell proliferation and oxidative stress [24]. TMSB10 and EPS8 were reported to promote proliferation and invasion of cancer cells $[25,26]$. In HTN samples, mesangial cells highly expressed CLDN4, a functional tight junction gene participating in tumor cell proliferation [27]. Upregulation of the above genes might be involved in proliferation of mesangial cells in HTN. IFI27, which was expressed preferentially in podocytes, was reduced in IgA nephropathy and membranous nephropathy patients and served as a useful genetic diagnostic marker [28]. Interestingly, we found upregulated IFI27 expression in mesangial cells from HTN subjects compared to the control samples. As expected, we observed upregulated inflammatory signatures in HTN mesangial cells compared to the control samples. CXCL14 functioned to recruit activated macrophages and was overexpressed in HTN mesangial cells. CD74 expression was also increased in mesangial cells of HTN subjects. It has been shown that increased level of endothelial CD74 mediates migration of macrophages and adhesion of monocytes [29]. SPP1, a typical inflammatory mediator [30]. was highly expressed in HTN mesangial cells. Furthermore, we explored functional differences between HTN and healthy mesangial cells by GO and KEGG analysis. DEGs in mesangial cells from HTN patients were enriched in leukocyte transendothelial migration, focal adhesion associated biological processes and negative regulation of MAPK cascade (Fig. 2d and 2e, Supplemental dataset. 5 and 6). These findings suggest that mesangial cells can produce 
inflammatory cytokines and chemokines which may build connections between mesangial cells and immune cells in kidneys, driving inflammatory reactions contributing to the development of HTN.

Pericytes, pluripotent perivascular cells closely related with endothelial maturation and vessel stability, have emerged as key players in the progression of hypertension and related organ damage [31]. Accordingly, we identified DEGs in renal pericytes by comparing HTN to the control subjects. Renal pericytes displayed down-regulated AMOTL1 expression, which was essential for endothelial/pericyte junctional complex formation and normal establishment of vascular networks [32]. This result indicates that altered gene expression in renal pericytes may contribute to endothelial dysfunction in HTN.

\section{HTN tubular cells were enriched with inflammation and oxidation related signatures}

Tubular epithelial cells were sub-grouped into 5 groups according to cell-cluster-specifically expressed genes. Molecular expression differences in tubular epithelial cells were compared between HTN and normal subjects, and DEGs were shown in Fig. 3a and Supplemental dataset. 3. We also compared cell signaling networks between HTN and control tubular cells. Proximal tubule cells displayed overexpression of genes enriched in PPAR signaling (Fig. 3b), response to oxidative stress and granulocyte activation involved in the immune response [33]. Genes participating in leukocyte transendothelial migration, IFN- $\gamma$-mediated signaling, toll-like receptor (TLR) signaling and regulation of chemokine IL-8/CXCL8 secretion, were increased in distal tubular cells (Fig. 3c). Furthermore, comparison of DEGs in loop of Henle cells showed enrichment of genes participating in NOD-like receptor signaling, TLR signaling, IL-17 signaling and IFN- $\gamma$-mediated signaling in HTN (Fig. 3d). As shown in Fig. 3e and 3f, genes involved in IFN- $\gamma$ signaling and cell redox homeostasis were increased both in principal cells and intercalated cells from HTN patients. Oxidation related signatures upregulated by tubular cells might contribute to oxidative stress in HTN. Therefore, overexpressed molecules involved in diverse inflammatory signaling pathways in tubules suggest tubular cells may serve as a main actor participating in inflammatory responses locally and systematically in the process of HTN. 


\section{Immune dysregulation participated in HTN progression}

Mounting evidence indicates that accumulation of $\mathrm{T}$ cells and monocytes/ macrophages in the renal interstitium has a prominent role in the generation and propagation of hypertension and target organ damage [34]. We detected nine immune cell subtypes infiltrating in kidneys of HTN patients, including NKT cell, NK cell, T cell, B cell and plasma cell (Fig. 4). Because of the limited number of leukocytes in the control kidney, HTN kidneys were compared with two peripheral blood mononuclear cell (PBMC) datasets from publicly available data [35,36]. For dendritic cells, upregulated genes were enriched in IFN- $\gamma$-mediated signaling, $\mathrm{T}$ cell costimulation and differentiation, and positive regulation of cell-cell adhesion in HTN (Fig. 5a, 5b). Interestingly, resident dendritic cells highly expressed several ribosomal-protein genes, which have recently been found to take part in the pathogenesis of hypertension [37].

As shown in Fig. 5c-h and Supplemental dataset. 4, DEGs were identified in infiltrating $\mathrm{T}$ cells including naïve $\mathrm{T}$ cell, $\mathrm{CD} 8^{+} \mathrm{MAIT}$ and $\mathrm{CD} 8^{+}$effector $\mathrm{T}$ cell from HTN kidneys compared to control PBMCs. Especially, chemokines and receptors including CXCL2, CXCL14, CCL3L1, CCRL2 and CCR2 were overexpressed in HTN effector $\mathrm{CD}^{+} \mathrm{T}$ cells, promoting the recruitment and migration of leukocytes to the renal interstitium. HTN effector $\mathrm{CD}^{+}$cells were also observed to express higher levels of inflammatory mediators such as SPP1, TNFAIP2 and TNFRSF9. For the HTN group, the GO terms of effector $\mathrm{CD}^{+} \mathrm{T}$ cells were enriched in $\mathrm{T}$ cell migration, proliferation, regulation of ubiquitin-protein transferase activity and inflammatory response. Of note, comparison of $\mathrm{CD}^{+}$MAIT from HTN and control subjects showed upregulation of genes participating in nuclear factor kappa-B (NF- $\mathrm{B}$ ) signaling, leukocyte chemotaxis, cell-matrix adhesion, oxidative stress and cellular response to vascular endothelial growth factor stimulus, indicating CD8 ${ }^{+}$MAIT might be an important contributor to the pathophysiology of hypertension. Hence, our findings suggest that $\mathrm{CD} 8^{+} \mathrm{T}$ cells may contribute to immune cell recruitment and migration and inflammatory cytokine secretion in HTN.

Next, we focused on upregulation of DEGs in plasma cells, and detected the infiltration of IGHG1-expressing plasma cells. Plasma cells in HTN patients had increased genes participating in complement activation, TNF-mediated signaling, endoplasmic reticulum stress, oxidative stress and regulation of protein ubiquitination compared to controls. 


\section{Ligand-receptor analysis revealed increased cell-cell crosstalk in HTN}

To explore cell-cell interactions in kidneys with HTN, ligand-receptor pair expression between distinct cell types were defined. We identified interactions of receptors and ligands and predicted intercellular communication for each cell cluster within the kidney (Fig. 6a, Supplemental dataset. 7). The intercellular interactions were primarily involved in mesangial cells, endothelial cells, fibroblasts, macrophages, monocytes, dendritic cells, loop of Henle cells and cycling epithelial cells, with less enrichment in plasma cells, T cells, B cells, principle cells, intercalated cells and podocytes.

Next, we determined the interplay between mesangial cells and other kidney cells such as endothelial cells, podocytes, fibroblasts, loop of Henle cells, monocytes, macrophages and cycling epithelial cells (Fig. 6b-e). The interacting pairs of ligands from mesangial cells and receptors from endothelial cells were also analyzed. Among them, the mesangial-endothelial cell-cell interaction was important. We found mesangial cells expressed chemokines including CXCL1, CCL2 and CXCL8, which all interacted with the chemokine receptor $A C K R 1$ expressed in the endothelium, indicating the chemoattractant ability for immune cells. Our network analysis revealed mesangial cell-derived ligand CD24 or GLG1 interacted with adhesion molecule SELP or SELE expressed in endothelial cells, which mediated the initial adhesion between leukocytes and resident glomerular cells. Furthermore, mesangial cells expressed $F G F 2$, a profibrotic factor related with ECM accumulation [38], its receptor, FGFR1, was detected in the endothelium, podocytes and fibroblasts. Mesangial cells also expressed TNF superfamily member TNFSF10, and its receptor, TNFRSF10A, was expressed in monocytes. The above results indicate that mesangial cells are involved in cell proliferation, ECM accumulation and inflammation in HTN. As illustrated in Fig. 6f-i, except for mesangial cells, the endothelium also displayed crosstalk with other cells in the kidney such as fibroblasts, cycling epithelial cells, macrophages, monocytes and dendritic cells. The expression of chemokines in the endothelium such as CCL2, CCL5 and CCL23 interacted with receptors CCR2 and CCR1 expressed in macrophages. Endothelial cells also expressed ACKR1, and its ligands CXCL1, CCL2, CCL5 and CXCL8 were expressed in macrophages, loop of Henle cells or cycling epithelial cells. Interestingly, CXCL12 and CCL5 expressed in the endothelium interacted with fibroblasts expressing ACKR3 and ACKR4, 
respectively. Endothelial cells expressed $P D G F D$ and $V E G F C$, which accelerated matrix-accumulation and angiogenesis by interacting with $P D G F R B$ and $K D R$ respectively expressed in fibroblasts (Fig. 4c). These findings support the key role of endothelial cells in immune cell infiltration, inflammation and fibrosis in HTN.

As we know, activation of Notch signaling plays a crucial role in angiogenesis, inflammation and fibrosis during development of HTN and renal fibrosis [39]. Particularly, our results showed JAG1/Notch signaling was detected comprehensively in different cell type interactions. The ligand $J A G 1$ expressed in mesangial cells or endothelial cells interacted with Notch receptors (NOTCH 1-4) expressed in various cells. Therefore, our data suggest that extensive intercellular crosstalk between the mesangial cells/endothelium and other cells in kidney may contribute to the progression of HTN.

\section{Discussion}

Here, we successfully generate a comprehensive single-cell transcriptome dataset for human HTN by scRNA-seq. To our knowledge, the current research is the first study to provide transcriptional landscapes of kidney samples in HTN patients by scRNA-seq analysis. Single cell transcriptomics for several human kidney diseases by scRNA-seq have been reported, while most of them focus on renal tubular epithelium and interstitium, and rarely assessed resident cells in glomerulus [14]. In this work, we improved the isolation method and identified all major kidney cell clusters including nephron and resident immune cells. Through comparing cell-type-specific gene expression and signaling signatures in kidneys from HTN biopsy specimens and living donor controls, our study reveals many meaningful results and provides clues for future research in the pathogenesis of HTN.

18 separate cell clusters were identified in the kidney, including three glomerular resident cell types, five tubular cell types, six types of immune cells, fibroblasts, smooth muscle cells, cycling epithelial cells and pericytes. Among them, T cells were further classified into several subclasses such as $\mathrm{CD}^{+}$naïve $\mathrm{T}$ cells, $\mathrm{CD} 8^{+} \mathrm{MAIT}$, $\mathrm{CD}^{+}$effector $\mathrm{T}$ cells and Tregs. Pericytes, specialized perivascular cells, have recently been considered as central regulators of endothelial cell dysfunction and microcirculation in the pathogenesis of hypertension [31]. Especially, AMOTL1 which was essential for normal vascular development and endothelial-pericyte crosstalk [32], 
was reduced in renal pericytes. Focusing on pericytes as a novel therapeutic target may ameliorate endothelial dysfunction and stabilize microcirculation in HTN.

The classical pathology of HTN contains nephroangiosclerosis and hyalinosis, structural remodeling and thickening of arterioles associates with hemodynamic alterations [40]. Emerging studies have revealed that endothelial dysfunction, vascular inflammation and angiogenesis participate in hypertension and targeted organ damage [41]. Our work showed that endothelial cells in HTN kidney exhibited different transcriptome phenotypes compared to controls. We detected enhanced LRG1 expression at the transcriptomic levels in endothelium of HTN patients. Evidence to date suggests that $L R G 1$ is associated with inflammatory responses, neutrophil differentiation and cell apoptosis [20]. LRG1 also served as a crucial pathogenic mediator in diabetic kidney diseases progression through TGF- $\beta$ /ALK1 signaling [19]. Interestingly, with regard to cardiovascular diseases, LRG was defined as a serum biomarker that discerns patients with heart failure accurately [42]. Thereby, we uncover the potential pathogenic effect of $L R G 1$ secreted by endothelial cells in pathogenesis of HTN. However, further research is needed to confirm whether $L R G 1$ emerges as a novel therapeutic target in hypertension and renal injury.

We also assessed the expression of other genes in endothelial cells from HTN kidneys except LRG1. Several upregulated cellular adhesion genes (SELP, CEACAMI and PECAM1) were demonstrated in endothelial cells from HTN subjects. Further, the endothelium overexpressed chemokines (CCL23 and CCL2) and chemokine receptor 1 (ACKRI). The highly expressed $V W A 1$ and $L Y V E-1$ in endothelium were associated with collagen accumulation and fibrosis [22,23]. Increased DEGs in the endothelium participated in TNF signaling, cellular response to IFN- $\gamma$, focal adhesion and cell-substrate adhesion. These data reflect HTN endothelium as a proinflammatory and profibrotic phenotype. Therefore, the endothelium may participate in trafficking and recruitment of immune cells, inflammatory reaction, and ECM accumulation during the process of hypertension.

Mesangial cell proliferation and mesangial expansion contribute to the development of HTN $[43,44]$. HTN mesangial cells overexpressd proliferation related signatures (MGST1, TMSB10, EPS8 and IER2) not detected in renal diseases before. We also showed upregulated genes (CXCL14, CD74 and SPP1), related with leukocyte migration and inflammation $[30,45,46]$. were also identified in mesangial cells of HTN patients. Thus, genes characteristic of HTN mesangium linked to 
mesangial cell proliferation and interaction with immune cells. As we know, tubulointerstitial inflammation and fibrosis are common characteristics of chronic renal disease progression, and renal prognosis exhibits a closer connection with the degree of tubulointerstitial damage than glomerular lesions [47]. Renal tubular epithelial cells play a pivotal role in the development of hypertension-induced renal damage [48]. In recent years, investigators have defined an important role of inflammatory pathways including IFN- $\gamma$ signaling and IL-17 signaling in several experimental hypertensive models, which promote both renal and vascular injury. IFN- $\gamma$ or IL-17A ablation results in blunted hypertension and does not exhibit endothelial dysfunction and target organ damage in animal models of hypertension $[49,50]$. Consistently, our results revealed that tubular cells obtained from HTN patients highly expressed genes involved in the IFN- $\gamma$ response signature and IL-17 signaling regulation. Furthermore, distal tubular cells and loop of Henle cells from HTN subjects also displayed upregulated TLR signature. Activation of TLR can activate downstream pathways including NF- $\kappa \mathrm{B}$ and MAPKs, and promote cytokine and chemokine production, participating in inflammatory processes of hypertension-induced target-organ damage [51]. These results define the renal epithelium as an important promoter contributing to inflammatory responses in the hypertension-challenged kidney.

Evidence from our group and others have revealed that both innate and adaptive immunity contribute to the pathogenesis of hypertension, primarily $\mathrm{T}$ cells and monocytes/macrophages accumulating in the kidney and vasculature [34]. We detected an elevated number of dendritic cells, macrophages, monocytes, $\mathrm{T}$ cells, $\mathrm{B}$ cells and plasma cells infiltrated in hypertensive kidneys. Our data indicated infiltrating HTN dendritic cells expressed markers enriched in IFN- $\gamma$-mediated signaling, $\mathrm{T}$ cell costimulation and differentiation, and cell-cell adhesion. Studies showed that $\mathrm{CD}^{-/}$mice displayed a blunted hypertension, and adoptive transfer of $\mathrm{CD}^{+}$but not $\mathrm{CD} 4^{+} \mathrm{T}$ cells caused hypertension inRAG- $1^{-/-}$mice with angiotensin II infusion, implying an unique prohypertensive effect of $\mathrm{CD} 8^{+} \mathrm{T}$ cells [52]. Accordingly, we identified 3 subpopulations of $\mathrm{CD}^{+} \mathrm{T}$ cells including $\mathrm{CD} 8^{+}$naïve $\mathrm{T}$ cells, $\mathrm{CD} 8^{+}$ MAIT and $\mathrm{CD}^{+}$effector $\mathrm{T}$ cells by cell cluster specific markers in HTN patients. Especially, HTN effector $\mathrm{CD}^{+} \mathrm{T}$ cells overexpressed chemokines and receptors including CXCL2, CXCL14, CCL3L1, CCRL2 and CCR2. CD8 ${ }^{+}$cells with effector phenotype also highly expressed inflammatory mediators including SPP1, TNFAIP2 
and TNFRSF9. Our novel findings indicate that HTN kidney exhibits an increased fraction of proinflammatory, chemotactic and cytotoxic $\mathrm{CD}^{+} \mathrm{T}$ cells.

To explore global cell-cell communications in HTN, ligand-receptor signaling among distinct cell populations was analyzed. Our observation showed that cell-type-specific interactions in HTN were most obvious in the endothelium or mesangial cells with other kidney-resident cells. It is well documented that inflammatory chemokines and their receptors account for increased immune cell infiltration and aggravated oxidative stress, vascular dysfunction, and fibrosis in hypertension [53-55]. Consistently, leukocyte chemotactic signaling and fibrotic related pathway were observed in ligand-receptor interactions across kidney cell types. Endothelial cells expressed the chemokine receptor $A C K R 1$, and its ligands CXCL1, CCL2, CCL5 and CXCL8 were expressed in mesangial cells, macrophages, loop of Henle cells and cycling epithelial cells. The endothelium also expressed chemokines including CCL2, CCL5 and CCL23, interacting with receptors CCR2 and CCR1 detected in macrophages. CXCL12 and CCL5 from the endothelium interacted with $A C K R 3$ and $A C K R 4$, respectively from fibroblasts. Endothelial cells also expressed $P D G F D$ and $V E G F C$, which accelerated matrix-accumulation by interacting with $P D G F R B$ and $K D R$, respectively in fibroblasts. Additionally, mesangial cells expressed profibrotic factor $F G F 2$, and genes encoding FGFRs were highly expressed in the endothelium, podocytes and fibroblasts. Previous studies have suggested that Notch signaling exhibited a pathogenic role in the progression of renal fibrosis [39]. Consistently, we found that intercellular interactions among diverse cells were enriched in JAG1/NOTCH signaling. It is anticipated that cell-cell crosstalk in the kidney contributes to the recruitment and infiltration of inflammatory cells into the kidneys, and fibrotic process, indicating the potential of utilizing novel molecular targets in hypertensive renal damage.

There were several limitations in our research. First, the number of patients was relatively small in the present study. Hence, increasing number of specimens is required in future study to reduce individual discrepancy, and reflect the severity of disease development in hypertensive renal damage. Second, despite the findings of novel cell-type-specific genes, the number of cells contained within a few cell clusters such as podocytes and macrophages was limited, which might not precisely reflect the transcriptomic status of all cell populations. Third, further functional analysis should be conducted to validate our scRNA-seq data in the future. 


\title{
Conclusions
}

In conclusion, despite the complexity and heterogeneity of HTN, our work suggests that scRNA-seq based approach is powerful and feasible in research of this field. We present comprehensive cell-specific transcriptome data of the HTN kidney, we also define novel hub genes and signaling pathways, exploring the molecular signatures relevant to the pathogenesis of HTN. Furthermore, we identify diverse intercellular communications which were predominantly observed between endothelial cells/mesangial cells with adjacent and remote cells in hypertensive renal damage. Our study may uncover new pathogenetic mechanisms and provide novel avenues for exploring new therapeutic targets for patients with hypertension and renal injury. These findings need to be further validated through in vitro and in vivo investigation.

\begin{abstract}
Abbreviations
HTN: Hypertensive nephropathy; scRNA-seq: single-cell RNA sequencing; CKD: chronic kidney diseases; RAAS: renin-angiotensin-aldosterone system; ACEI: angiotensin-converting enzyme inhibitors; ARB: angiotensin II receptor blockers; RNA-seq: RNA sequencing; eGFR: estimated glomerular filtration rate; DEGs: differentially expressed genes; GO: Gene Ontology; KEGG: Kyoto Encyclopedia of Genes and Genomes; ECM: extracellular matrix; PBMC: peripheral blood mononuclear cell; NF- к B: nuclear factor kappa-B.
\end{abstract}

\section{Authors' contributions}

Y.Z., R.T., W.L. and X.C.X. conceived and designed the experiments; Y.Z., R.T., J.D.O. and X.C.X. wrote the manuscript; J.D.O., P.J.E., X.A., W.S.P., Q.L.Z. and P.X. revised the manuscript; T.M., W.L., Y.Z. and W.N.N. performedthe experiments; R.T., T.M., W.L. and C.J.S generated and provided analytical tools; J.B.C., R.T., Y.Z., X.C.X., C.J.S, P.J. and X.D analyzed data; all theauthors approved the final version of the manuscript.

\section{Author details}

${ }^{1}$ Department of Nephrology, Xiangya Hospital, Central South University, Changsha, Hunan Province, China. ${ }^{2}$ Department of Pathology, Xiangya Hospital, Central South University, Changsha, Hunan Province, China. ${ }^{3}$ Department of Hematology, the Affiliated Zhuzhou Hospital Xiangya Medical College, Central South University, Zhuzhou, Hunan Province, China. ${ }^{4}$ Centre for Inflammatory Diseases, Monash University, Clayton, Victoria, 3168, Australia. ${ }^{5}$ Department of Organ Transplantation, Xiangya Hospital, Central South University, Changsha, China. 
${ }^{6}$ Department of Medical records \& information, Xiangya Hospital, Central South University, Changsha, Hunan Province, China.

\section{Acknowledgements}

The authors thank all the staff of the Department of Nephrology in Xiangya Hospital for their kindly assistance in collecting samples from patients.

\section{Competing interests}

All authors declared no potential competing interests associated with the manuscript.

\section{Consent for publication}

Not applicable.

\section{Data availability}

All datasets analysed to support the conclusions of this article are included within the article.

\section{Ethics approval and consent to participate}

These studies were approved by the Medical Ethics Committee of Xiangya Hospital of Central South University for Human Studies (No. 201711836). All subjects needed written informed consent.

\section{Funding}

This work was funded by the National Key R\&D Program of China (2020YFC2005000 to XX), the National Natural Science Foundation of China (81800641 to TM and 81500559 to RT), the Key Research and Development Program of Hunan province (2018WK2060 to XX and 2020WK2008 to YZ), the science and technology innovation Program of Hunan Province (2020RC5002 to JO), the Natural Science Foundation of Hunan Province (2018JJ3818 to RT and 2019JJ40515 to WNN), Chinese Society of Nephrology (18020010780 to YZ).

\section{References}

1. Messerli FH, Williams B, Ritz E. Essential hypertension. Lancet. 2007;370:591-603.

2. Kearney PM, Whelton M, Reynolds $\mathrm{K}$ et al. Global burden of hypertension: analysis of worldwide data. Lancet. 2005;365:217-23.

3. Udani S, Lazich I, Bakris GL. Epidemiology of hypertensive kidney disease. Nat Rev Nephrol. 2011;7:11-21.

4. Ruiz-Ortega M, Rayego-Mateos S, Lamas S, et al. Targeting the progression of chronic kidney disease. Nat Rev Nephrol. 2020;16:269-88.

5. Leiba A, Fishman B, Twig G, et al. Association of Adolescent Hypertension With Future End-stage Renal Disease. JAMA Intern Med. 2019;179:517-23. 
6. Griffin KA. Hypertensive Kidney Injury and the Progression of Chronic Kidney Disease. Hypertension. 2017;70:687-94.

7. Johnson RJ, Segal MS, Srinivas T, et al. Essential hypertension, progressive renal disease, and uric acid: a pathogenetic link? J Am Soc Nephrol. 2005;16:1909-19.

8. Seccia TM, Caroccia B, Calò LA. Hypertensive nephropathy. Moving from classic to emerging pathogenetic mechanisms. J Hypertens. 2017;35:205-12.

9. Bidani AK, Griffin KA. Pathophysiology of hypertensive renal damage: implications for therapy. Hypertension. 2004;44:595-601.

10. Svensson V, Natarajan KN, Ly LH, et al. Power analysis of single-cell RNA-sequencing experiments. Nat Methods. 2017;14(4):381-7.

11. Wang Z, Gerstein M, Snyder M. RNA-Seq: a revolutionary tool for transcriptomics. Nat Rev Genet. 2009;10:57-63.

12. Huang B, Chen Z, Geng L, et al. Mucosal Profiling of Pediatric-Onset Colitis and IBD Reveals Common Pathogenics and Therapeutic Pathways. Cell. 2019;179:1160-76.

13. Patel AP, Tirosh I, Trombetta JJ, et al. Single-cell RNA-seq highlights intratumoral heterogeneity in primary glioblastoma. Science. 2014;344:1396-401.

14. Der E, Suryawanshi H, Morozov P, et al. Accelerating Medicines Partnership Rheumatoid Arthritis and Systemic Lupus Erythematosus (AMP RA/SLE) Consortium. Tubular cell and keratinocyte single-cell transcriptomics applied to lupus nephritis reveal type I IFN and fibrosis relevant pathways. Nat Immunol. 2019;20:915-27.

15. Wilson PC, Wu H, Kirita Y, et al. The single-cell transcriptomic landscape of early human diabetic nephropathy. Proc Natl Acad Sci U S A. 2019;116:19619-25.

16. Chokshi SM, Ma Q, Drake KA, et al. Single-Cell Profiling of AKI in a Murine Model Reveals Novel Transcriptional Signatures, Profibrotic Phenotype, and Epithelial-to-Stromal Crosstalk. J Am Soc Nephrol. 2020;31:2793-814.

17. Zheng Y, Lu P, Deng Y, et al. Single-Cell Transcriptomics Reveal Immune Mechanisms of the Onset and Progression of IgA Nephropathy. Cell Rep. 2020;33:108525.

18. Li M, Liu H, Guo Y, et al. Single symbioticcell transcriptome sequencing of coral. Genomics. 2020;112:5305-12.

19. Hong Q, Zhang L, Fu J, et al. LRG1 Promotes Diabetic Kidney Disease Progression by Enhancing TGF- $\beta$-Induced Angiogenesis. J Am Soc Nephrol. 2019;30:546-62.

20. Jin J, Sun H, Liu D, et al. LRG1 Promotes Apoptosis andAutophagythrough the TGF $\beta$-smad1/5 Signaling Pathway to Exacerbate Ischemia/Reperfusion Injury. Neuroscience. 2019;413:123-34.

21. Girbl T, Lenn T, Perez L, et al. Distinct Compartmentalization of the Chemokines CXCL1 and CXCL2 and the Atypical Receptor ACKR1 Determine Discrete Stages of Neutrophil Diapedesis. Immunity. 2018;49:1062-1076.e6.

22. Fitzgerald J. WARP: A Unique Extracellular Matrix Component of Cartilage, Muscle, and Endothelial Cell Basement Membranes. Anat Rec (Hoboken). 2020;303:1619-23.

23. Lim HY, Lim SY, Tan CK, et al. Hyaluronan Receptor LYVE-1-Expressing Macrophages Maintain Arterial Tone through Hyaluronan-Mediated Regulation of Smooth Muscle Cell Collagen. Immunity. 2018;49:326-41.e7.

24. Zeng B, Ge C, Li R, et al. Knockdown ofmicrosomal glutathione S-transferase 1 inhibits lung adenocarcinoma cell proliferation and induces apoptosis. Biomed Pharmacother. 
2020;121:109562.

25. Zeng J, Yang X, Yang L, et al. Thymosin $\beta 10$ promotes tumor-associatedmacrophages M2 conversion and proliferation via the PI3K/Akt pathway in lung adenocarcinoma. Respir Res. 2020;21:328.

26. Maa MC, Lee JC, Chen YJ, et al. Eps8 facilitates cellular growthand motility of colon cancer cells by increasing the expression and activity of focal adhesion kinase. J Biol Chem. 2007;282:19399-409.

27. Song YX, Sun JX, Zhao JH, et al. Non-coding RNAs participate in the regulatory network of CLDN4 via ceRNA mediated miRNA evasion. Nat Commun. 2017;8:289.

28. Nagasawa Y, Okuzaki D, Muso E, et al. IFI27 Is a Useful Genetic Marker for Diagnosis of Immunoglobulin A Nephropathy and Membranous Nephropathy Using Peripheral Blood. PLoS One. 2016;11:e0153252.

29. Bernhagen J, Krohn R, Lue H, et al. MIF is a noncognate ligand of CXC chemokine receptors in inflammatory and atherogenic cell recruitment. Nat Med. 2007;13:587-96.

30. Lamort AS, Giopanou I, Psallidas I, et al. Osteopontin as a Link between Inflammation and Cancer: The Thorax in the Spotlight. Cells. 2019;8:815.

31. Špiranec K, Chen W, Werner F, et al. Endothelial C-Type Natriuretic Peptide Acts on Pericytes to Regulate Microcirculatory Flow and Blood Pressure. Circulation. 2018;138:494-508.

32. Zheng Y, Vertuani S, Nyström S, et al. Angiomotin-like protein 1 controls endothelial polarity and junction stability during sprouting angiogenesis. Circ Res. 2009;105:260-70.

33. Vallée A, Lecarpentier Y. Crosstalk Between Peroxisome Proliferator-Activated Receptor Gamma and the Canonical WNT/ $\beta$-Catenin Pathway in Chronic Inflammation and Oxidative Stress During Carcinogenesis. Front Immunol. 2018;9:745.

34. McMaster WG, Kirabo A, Madhur MS, Harrison DG.Inflammation, immunity, and hypertensive end-organ damage. Circ Res. 2015;116:1022-33.

35. 10X-Genomics, Data from "3k PBMCs from a Healthy Donor." $10 \mathrm{X}$ Genomics. 2016.https://support.10xgenomics.com/single-cell-gene-expression/datasets/1.1.0/pbmc3k.Access ed 14 April 2019.

36. 10X-Genomics, Data from "4k PBMCs from a Healthy Donor." 10X Genomics. 2017.https://support.10xgenomics.com/single-cell-gene-expression/datasets/2.1.0/pbmc4k.Access ed 14 April 2019.

37. Marques FZ, Nelson E, Chu PY, et al. High-Fiber Diet and Acetate Supplementation Change the Gut Microbiota and Prevent the Development of Hypertension and Heart Failure in Hypertensive Mice. Circulation. 2017;135:964-77.

38. Livingston MJ, Ding HF, Huang S, et al. Persistent activation of autophagy in kidney tubular cells promotes renal interstitial fibrosis during unilateral ureteral obstruction. Autophagy. 2016;12:976-98.

39. Edeling M, Ragi G, Huang S, et al. Developmental signalling pathways in renal fibrosis: the roles of Notch, Wnt and Hedgehog. Nat Rev Nephrol. 2016;12:426-39.

40. Liang S, Le W, Liang D, et al. Clinico-pathological characteristics and outcomes of patients with biopsy-proven hypertensive nephrosclerosis: a retrospective cohort study. BMC Nephrol. 2016;17:42.

41. Freedman BI, Cohen AH. Hypertension-attributed nephropathy: what's in a name? Nat Rev Nephrol. 2016;12:27-36. 
42. Watson CJ, Ledwidge MT, Phelan D, et al. Proteomic analysis of coronary sinus serum reveals leucine-rich $\alpha 2$-glycoprotein as a novel biomarker of ventricular dysfunction and heart failure. Circ Heart Fail. 2011;4:188-97.

43. Berman S, Abu Hamad R, Efrati S. Mesangial cells are responsible for orchestrating the renal podocytes injury in the context of malignant hypertension. Nephrology (Carlton). 2013;18:292-8.

44. Efrati S, Berman S, Goldfinger N, et al. Enhanced angiotensin II production by renal mesangium is responsible for apoptosis/proliferation of endothelial and epithelial cells in a model of malignant hypertension. J Hypertens. 2007;25:1041-52.

45. Valiño-Rivas L, Baeza-Bermejillo C, Gonzalez-Lafuente L, et al. CD74 in Kidney Disease. Front Immunol. 2015;6:483.

46. Lu J, Chatterjee M, Schmid H, et al. CXCL14 as an emerging immune and inflammatory modulator. J Inflamm (Lond). 2016;13:1.

47. Liu Y. Cellular and molecular mechanisms of renal fibrosis. Nat Rev Nephrol. 2011;(18) 7:684-96.

48. Mennuni S, Rubattu S, Pierelli G, et al. Hypertension and kidneys: unraveling complex molecular mechanisms underlying hypertensive renal damage. J Hum Hypertens. 2014;28:74-9.

49. Markó L, Kvakan H, Park JK, et al. Interferon- $\gamma$ signaling inhibition ameliorates angiotensin II-induced cardiac damage. Hypertension. 2012;60:1430-6.

50. Madhur MS, Lob HE, McCann LA, et al. Interleukin17 promotesangiotensin II-induced hypertension and vascular dysfunction. Hypertension. 2010;55:500-7.

51. Singh MV, Abboud FM. Toll-like receptors and hypertension. Am J Physiol Regul Integr Comp Physiol. 2014;307:R501-4.

52. Trott DW, Thabet SR, Kirabo A, et al. Oligoclonal CD8+ T cells play a critical role in the development of hypertension. Hypertension. 2014;64:1108-15.

53. Mikolajczyk TP, Szczepaniak P, Vidler F, et al. Role of inflammatory chemokines in hypertension. Pharmacol Ther 2020;223:107799

54. Alsheikh AJ, Dasinger JH, Abais-Battad JM, et al. CCL2 mediates early renal leukocyte infiltration during salt-sensitive hypertension. Am J Physiol Renal Physiol. 2020;318:F982-93.

55. Shimizu K, Furuichi K, Sakai N, et al. Fractalkine and its receptor, CX3CR1, promote hypertensive interstitial fibrosis in the kidney. Hypertens Res. 2011;34:747-52.

\section{Figure legends}

Fig. 1 Distinct cells clusters in kidney identified by scRNA-seq technology in HTN and control subjects. (a) UMAP plot display eighteen distinct cell types in the kidney. Cells were colored by different subpopulations. (b) UMAP plot of cell clusters from a single individual with HTN ( $\mathrm{n}=2$ ) and control donor $(\mathrm{n}=2)$. The color of cells represents the individual origin. (c) Bar chart shows the proportion of cell types in the kidney from distinct subjects. Blocks reflect different subjects, cells are colored by different types, and the quantity of cells was proportional to the height of the blocks. (d) Heatmap showing the top 20 gene markers of each identified cluster. Each column was a single cell cluster, and each row showed differentially expressed marker genes. (e) Violin plots reveal selected lineage markers across eighteen cell clusters. The colors indicate a representative 
marker gene from each cell population. Abbreviations are as follows: PTC, proximal tubule cells; IC, intercalated cells; LOH, loop of Henle cells; PC, principal cells; DTC, distal tubule cells; MES, mesangial cells; DC, dendritic cells; MC, macrophages; MON, monocytes; POD, podocytes; EC, endothelial cells; PLA, plasma cells; B, B cells; T, T cells; FIB, fibroblasts; PER, pericytes; SMC, smooth muscle cells; Cycling Epi, cycling epithelial cells. NC, normal control;HT, hypertension patient.

Fig. 2 Differential gene expression and altered pathways in endothelial cells and mesangial cells from HTN and control subjects. (a) Representative DEGs in endothelial cells and mesangial cells between HTN and control subjects. GO terms of DEGs in hypertensive endothelial cells (b) and mesangial cells (d), respectively versus controls. KEGG enrichment analysis demonstrates that increased DEGs participate in diverse signaling pathways in hypertensive endothelial cells (c) )and mesangial cells (e) respectively in comparison with control subjects. Abbreviations: pct.exp, percentage of cell expressing gene; count, number of genes annotated to KEGG pathway or GO terms.

Fig. 3 Differential gene expression and altered pathways in tubules from HTN and control subjects. (a) Representative DEGs in proximal tubule cells, distal tubule cells, loop of Henle cells, principal cells and intercalated cells between HTN and control subjects. GO and KEGG enrichment analysis shows upregulated DEGs were involved in some biological functions and signaling pathways in proximal tubule cells (b), distal tubule cells (c), loop of Henle cells (d), principal cells (e), and intercalated cells (f), respectively comparing with controls. Abbreviations: pct.exp, percentage of cell expressing gene; count, number of genes annotated to KEGG pathway or GO terms.

Fig. 4 Classification of immune cells in kidneys from HTN and control subjects. (a) Immune cells from the kidney are separated into nine subtypes by tSNE plot. Cells are colored by different subpopulations. (b) tSNE plot of immune cell subgroups in kidney from single individual of HTN $(n=2)$ and control $(n=2)$. Cells are colored by individual origin. (c) Bar chart showing percentage of cell subtypes in the kidney from different subjects. Blocks represent different subjects, colors represent different cell subclusters, and cell number is proportional to block height.

Fig. 5 Differential gene expression and altered pathways in immune cells from HTN and control subjects. (a) Representative DEGs in dendritic cells, monocytes and macrophages between HTN and control subjects. (c) Representative DEGs in naïve $\mathrm{T}$ cells, $\mathrm{CD} 8^{+}$naïve $\mathrm{T}$ cells, $\mathrm{CD} 8^{+}$MAIT and $\mathrm{CD} 8^{+}$effector $\mathrm{T}$ cells and plasma cells between HTN and control subjects. GO and KEGG enrichment analysis show upregulated DEGs were involved in some biological functions and signaling pathways in dendritic cells $(\mathbf{b})$, naïve T cells $(\mathbf{d}), \mathrm{CD} 8^{+}$naïve T cells $(\mathbf{e}), \mathrm{CD} 8^{+}$MAIT (f) 
and $\mathrm{CD}^{+}$effector $\mathrm{T}$ cells $(\mathbf{g})$ and plasma cells (h) in comparison with controls respectively. Abbreviations: pct.exp, percentage of cell expressing gene; count, number of genes annotated to KEGG pathway or GO terms.

Fig. 6 Cell-cell crosstalk between different cell clusters in the kidney of HTN patients. (a) Cell-cell communication events in kidney by analysis of ligand-receptor interactions between distinct cell types are shown. Visualized graphs indicate representative ligand-receptor interactions between mesangial cells and endothelial cells (b), podocytes (c), fibroblasts (d), or monocytes (e). Representative ligand-receptor interactions between endothelial cells and macrophages (f), fibroblasts (g), loop of Henle cells (h), or cycling epithelial cells (i). Lines represent interrelations between the ligand and corresponding receptor. Genes shown in blue represent ligands, and those depicted in red are receptors. Only HTN patients $(n=2)$ were analyzed.

Table. 1 Cell-cluster-specific marker genes of different cell types.

\begin{tabular}{|l|l|}
\hline Cell type & Marker genes \\
\hline Melanesian cells (MES) & FHL2, FN1, MYL9 \\
\hline Endothelial cells (EC) & CDH5, PECAM1, KDR, CLDN5 \\
\hline Phagocytes(POD) & NPHS2, PODXL, PTPRO \\
\hline Proximal tubular cells(PT) & CUBN, SLC13A1, LRP2, ALDOB \\
\hline Distal tubular cells(DT) & CALB1, SLC12A3 \\
\hline Loop of Helen cells(LOH) & UMOD, SLC12A1, CLDN16 \\
\hline Principal cells(PC) & AQP2, AQP3, GATA3 \\
\hline Intercalated cells(IC) & SLC4A1, ATP6V0D2, FOXI1, DMRT2 \\
\hline Macrophages(MC) & MRC1, CD68, CD163, C1QA \\
\hline Monocytes(MON) & LYZ, CD14, VCAN, FCN1 \\
\hline Dendritic cells(DC) & CD1C, FCER1A, CLEC10A, IRF8 \\
\hline T cells(T) & CD3D, CD3G, TRBC1 \\
\hline B cells(B) & MS4A1, CD79A, CD79B \\
\hline Plasma cells(PLA) & IGHG1, JCHAIN, MZB1 \\
\hline Fibroblasts(FIB) & COL1A1, DCN, LUM \\
\hline Smooth muscle cells(SMC) & ACTA2, TAGLN, MYLK, MYH11 \\
\hline Pericytes(PER) & RGS5, ACTA2, MCAM, PDGFRB \\
\hline $\begin{array}{l}\text { Cycling Epithelial cells } \\
\text { Cycling Epi) }\end{array}$ & TOP2A, EPCAM, CALB1, UMOD, CUBN \\
\hline
\end{tabular}




\section{Supplementary materials}

Supplemental Table. 1 Cell number, viability and gene information for each sample

Supplemental dataset. 1 Heatmap of the top 20 marker genes of each cell type

Supplemental dataset. 2 DEGs in endothelial and Melanesian cells comparing HTN with control subjects. Abbreviations: pct.1, the percentage of cells where the gene is determined in HTN group ; pct.2, the percentage of cells where the gene is investigated in control group; avg $\operatorname{logFC}, \log$ fold-chage of the average expression comparing the two groups, positive values indicate that the gene is expressed more highly in the first group.

Supplemental dataset. 3 DEGs in tubular cells comparing HTN with control subjects

Supplemental dataset. 4 DEGs in immune cells comparing HTN with control subjects

Supplemental dataset. 5 GO enrichment analysis of DEGs in different cell clusters from HTN and control subjects

Supplemental dataset. 6 KEGG enrichment analysis of DEGs in different cell clusters from HTN and control subjects

Supplemental dataset. 7 Putative ligand-receptor interactions between distinct cell clusters in kidney from HTN patients 


\section{Figures}

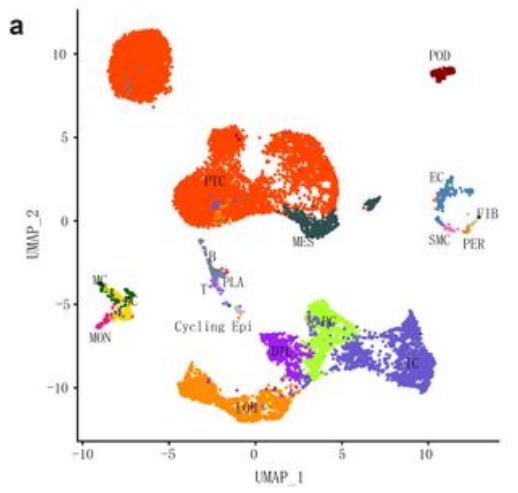

b

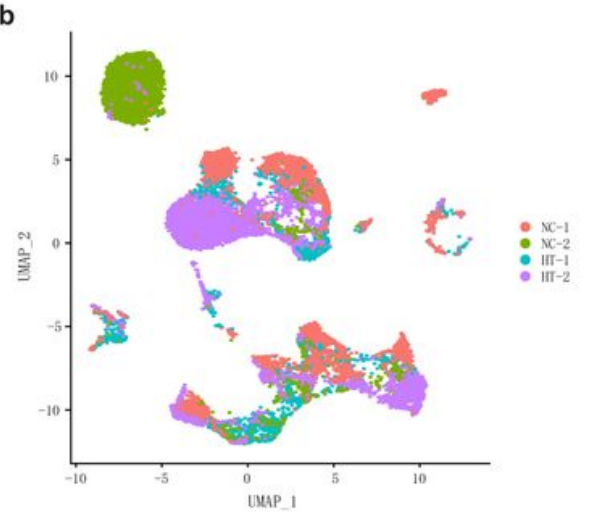

c

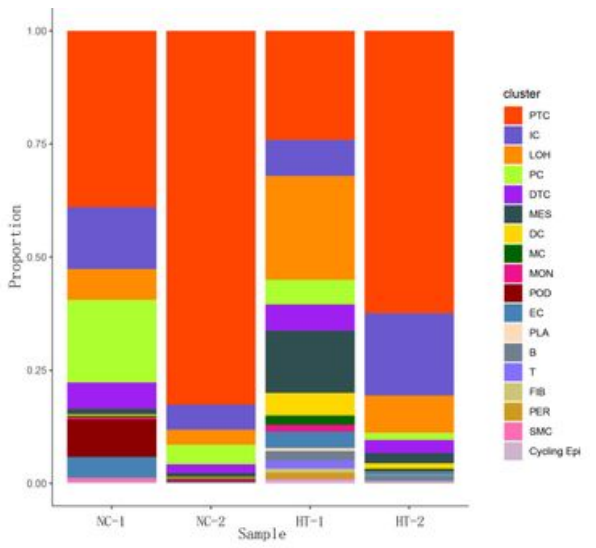

d
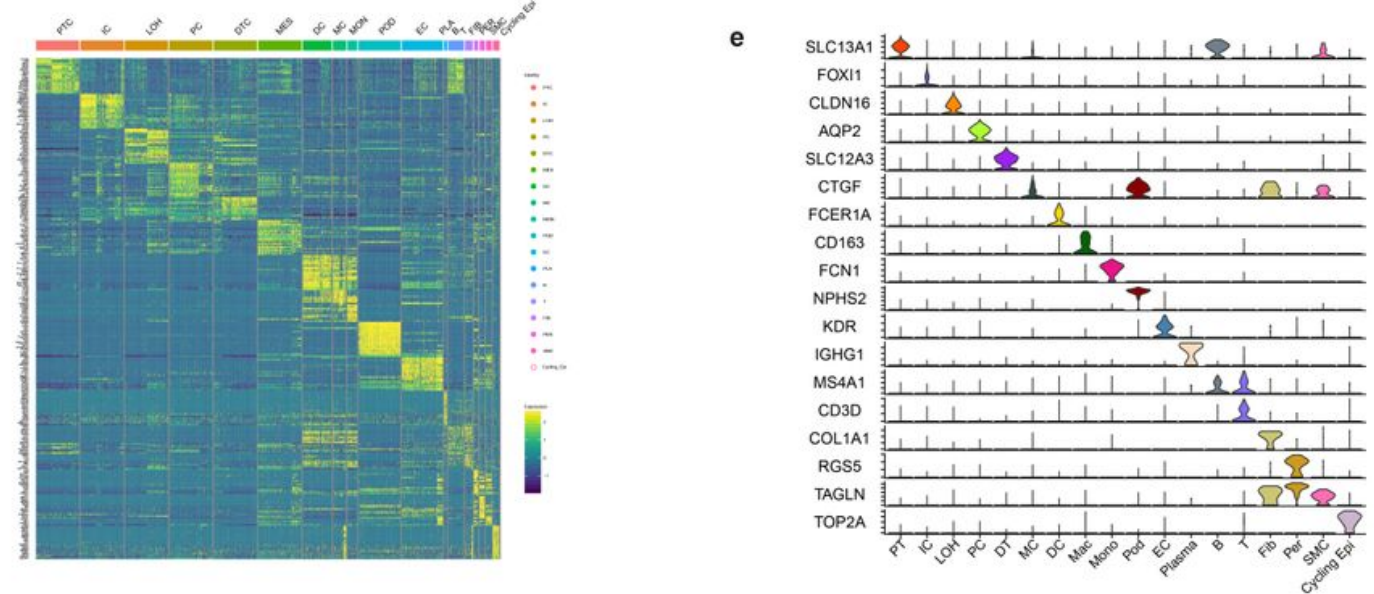

Figure 1

Distinct cells clusters in kidney identified by scRNA-seq technology in HTN and control subjects. (a) UMAP plot display eighteen distinct cell types in the kidney. Cells were colored by different subpopulations. (b) UMAP plot of cell clusters from a single individual with HTN $(n=2)$ and control donor $(n=2)$. The color of cells represents the individual origin. (c) Bar chart shows the proportion of cell types in the kidney from distinct subjects. Blocks reflect different subjects, cells are colored by different types, and the quantity of cells was proportional to the height of the blocks. (d) Heatmap showing the top 20 gene markers of each identified cluster. Each column was a single cell cluster, and each row showed differentially expressed marker genes. (e) Violin plots reveal selected lineage markers across eighteen cell clusters. The colors indicate a representative marker gene from each cell population. Abbreviations are as follows: PTC, proximal tubule cells; IC, intercalated cells; LOH, loop of Henle cells $\triangle P C$, principal cells; DTC, distal tubule cells; MES, mesangial cells; DC, dendritic cells; MC, macrophages; MON, monocytes; POD, podocytes; EC, endothelial cells; PLA, plasma cells; B, B cells; T, T cells; FIB, fibroblasts; PER, pericytes; $\mathrm{SMC}$, smooth muscle cells; Cycling Epi, cycling epithelial cells. NC, normal control;HT, hypertension patient. 


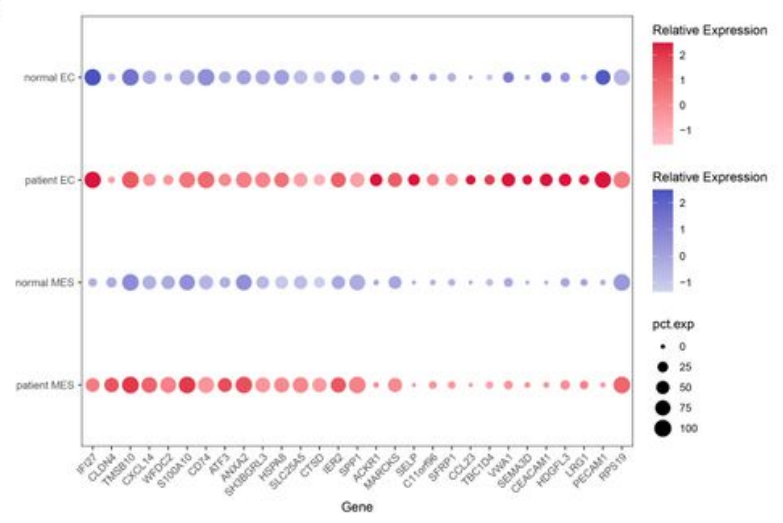

b

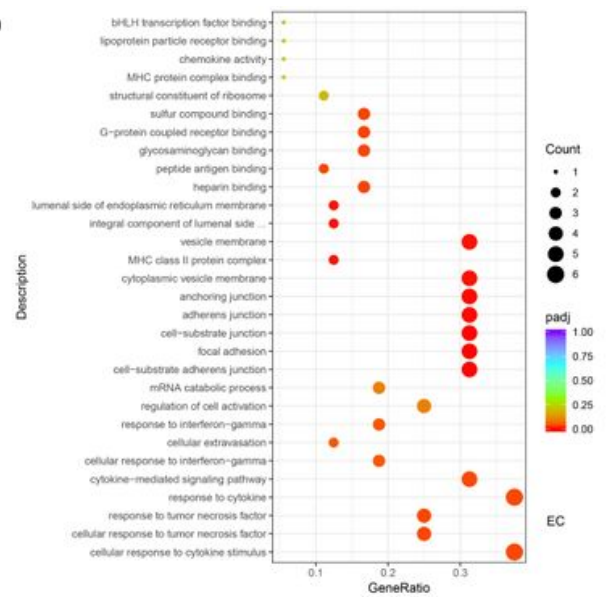

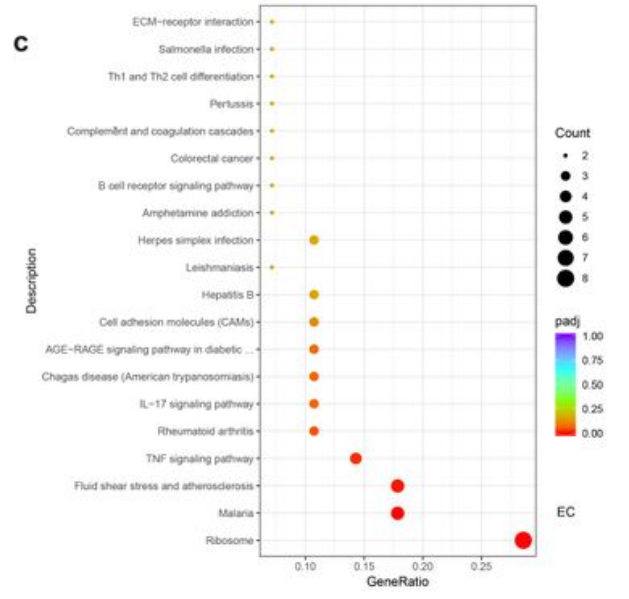
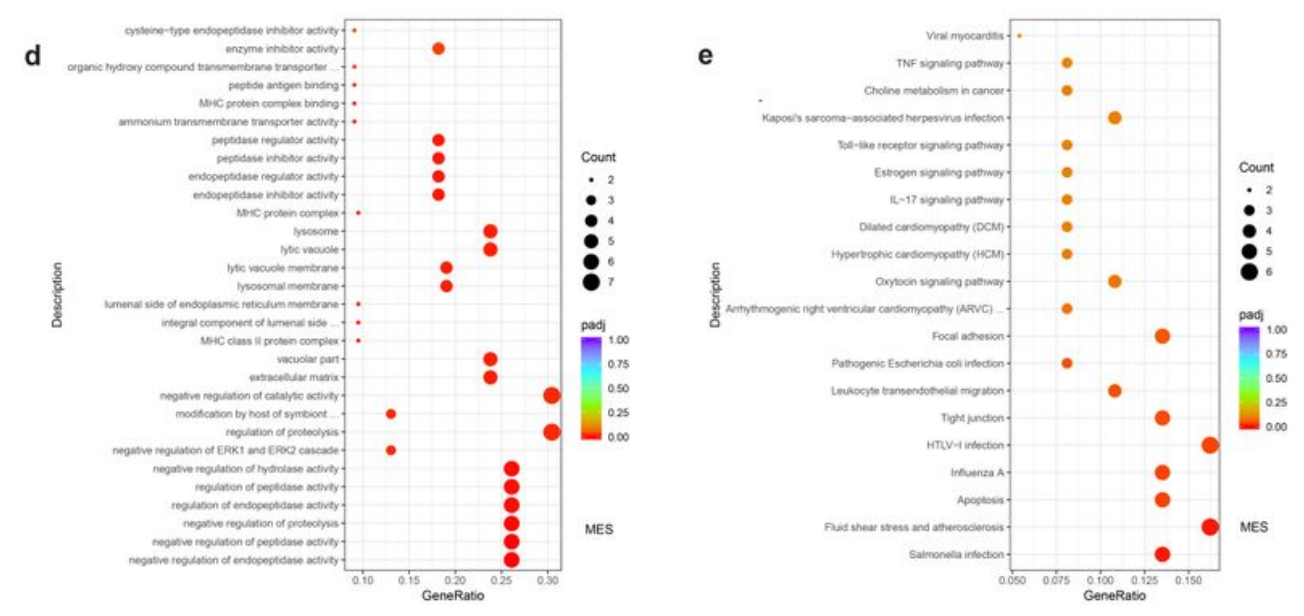

Figure 2

Differential gene expression and altered pathways in endothelial cells and mesangial cells from HTN and control subjects. (a) Representative DEGs in endothelial cells and mesangial cells between HTN and control subjects. GO terms of DEGs in hypertensive endothelial cells (b) and mesangial cells (d), respectively versus controls. KEGG enrichment analysis demonstrates that increased DEGs participate in diverse signaling pathways in hypertensive endothelial cells (c) )and mesangial cells (e) respectively in comparison with control subjects. Abbreviations: pct.exp, percentage of cell expressing gene; count, number of genes annotated to KEGG pathway or GO terms. 



Figure 3

Differential gene expression and altered pathways in tubules from HTN and control subjects. (a) Representative DEGs in proximal tubule cells, distal tubule cells, loop of Henle cells, principal cells and intercalated cells between HTN and control subjects. GO and KEGG enrichment analysis shows upregulated DEGs were involved in some biological functions and signaling pathways in proximal tubule cells (b), distal tubule cells (c), loop of Henle cells (d), principal cells (e), and intercalated cells (f), respectively comparing with controls. Abbreviations: pct.exp, percentage of cell expressing gene; count, number of genes annotated to KEGG pathway or GO terms. 
a

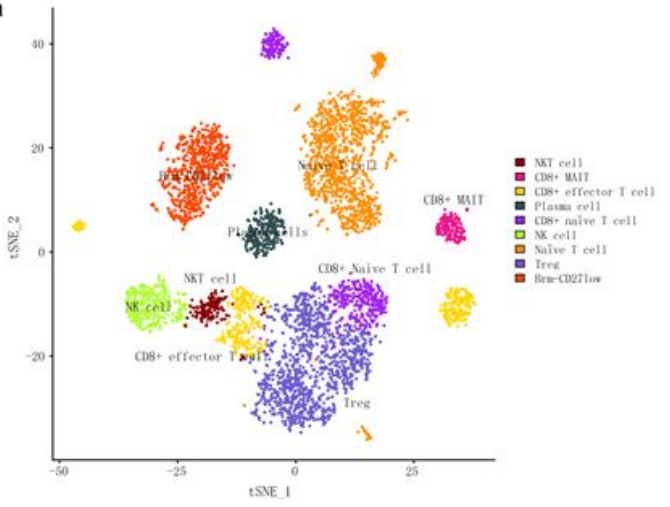

b

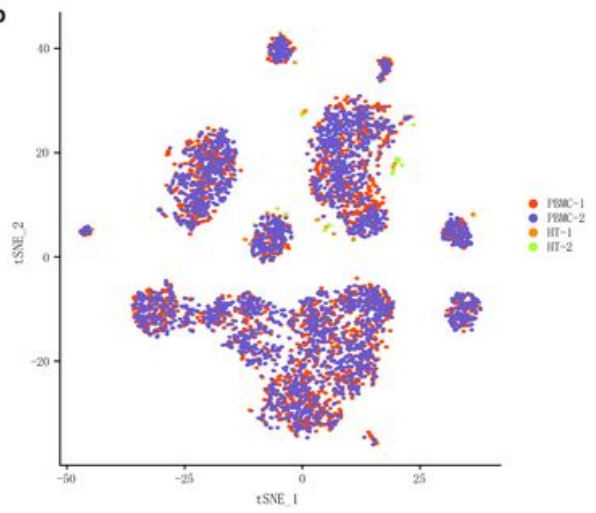

c

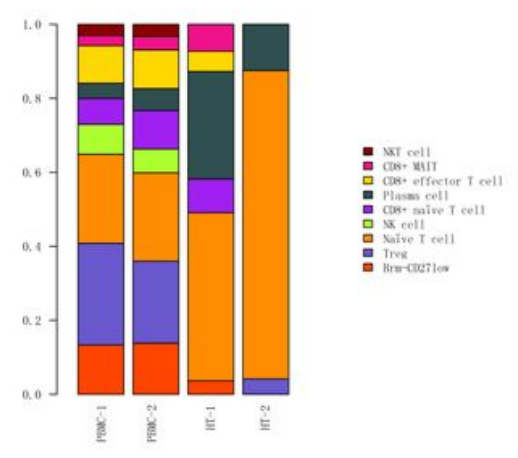

Figure 4

Classification of immune cells in kidneys from HTN and control subjects. (a) Immune cells from the kidney are separated into nine subtypes by tSNE plot. Cells are colored by different subpopulations. (b) tSNE plot of immune cell subgroups in kidney from single individual of HTN $(n=2)$ and control $(n=2)$. Cells are colored by individual origin. (c) Bar chart showing percentage of cell subtypes in the kidney from different subjects. Blocks represent different subjects, colors represent different cell subclusters, and cell number is proportional to block height. 

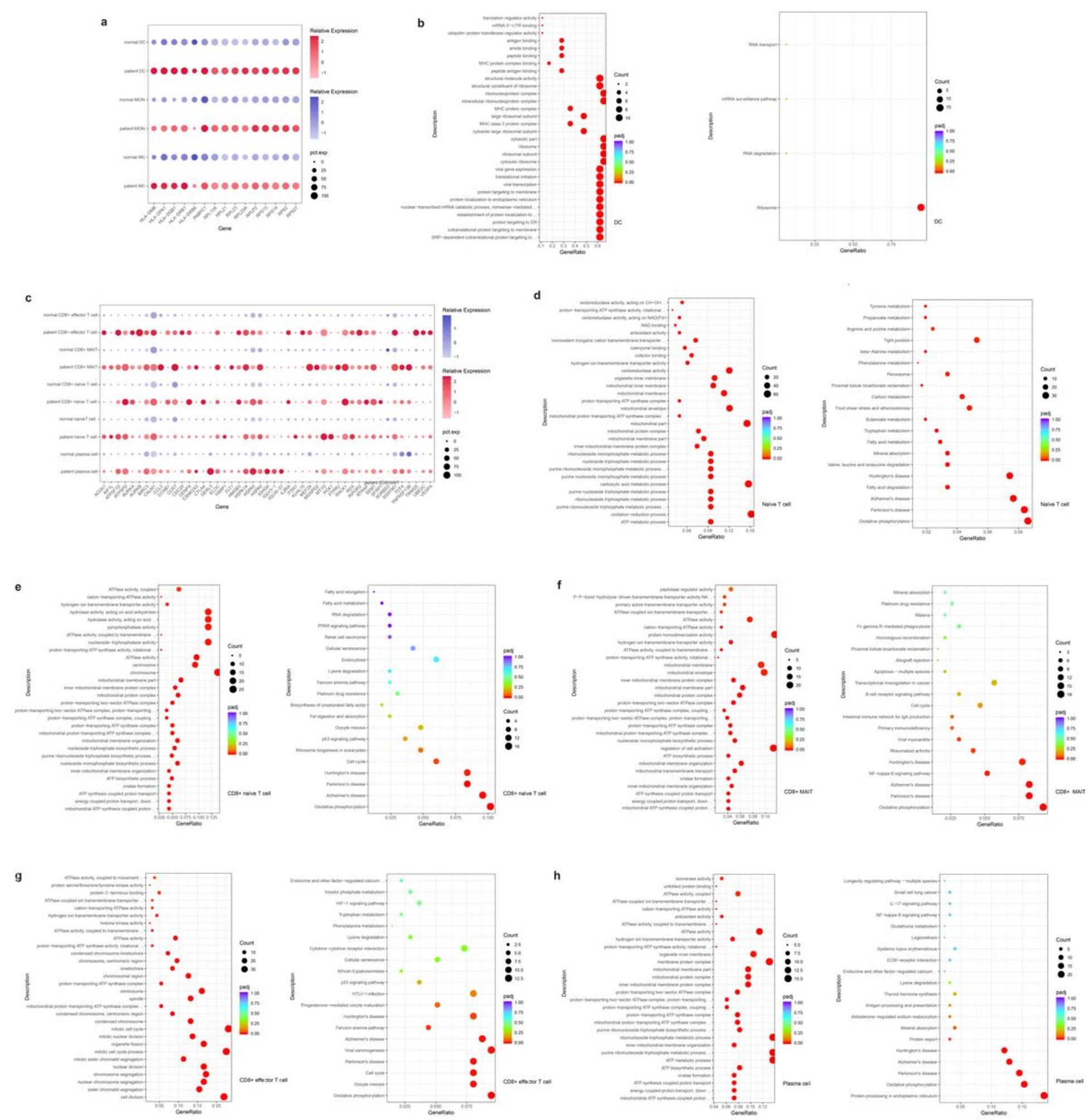

Figure 5

Differential gene expression and altered pathways in immune cells from HTN and control subjects. (a) Representative DEGs in dendritic cells, monocytes and macrophages between HTN and control subjects. (c) Representative DEGs in naïve T cells, CD8+ naïve T cells, CD8+ MAIT and CD8+ effector T cells and plasma cells between HTN and control subjects. GO and KEGG enrichment analysis show upregulated DEGs were involved in some biological functions and signaling pathways in dendritic cells (b), naïve T 
cells (d), CD8+ naïve T cells (e), CD8+ MAIT (f) and CD8+ effector T cells (g) and plasma cells (h) in comparison with controls respectively. Abbreviations: pct.exp, percentage of cell expressing gene; count, number of genes annotated to KEGG pathway or GO terms.
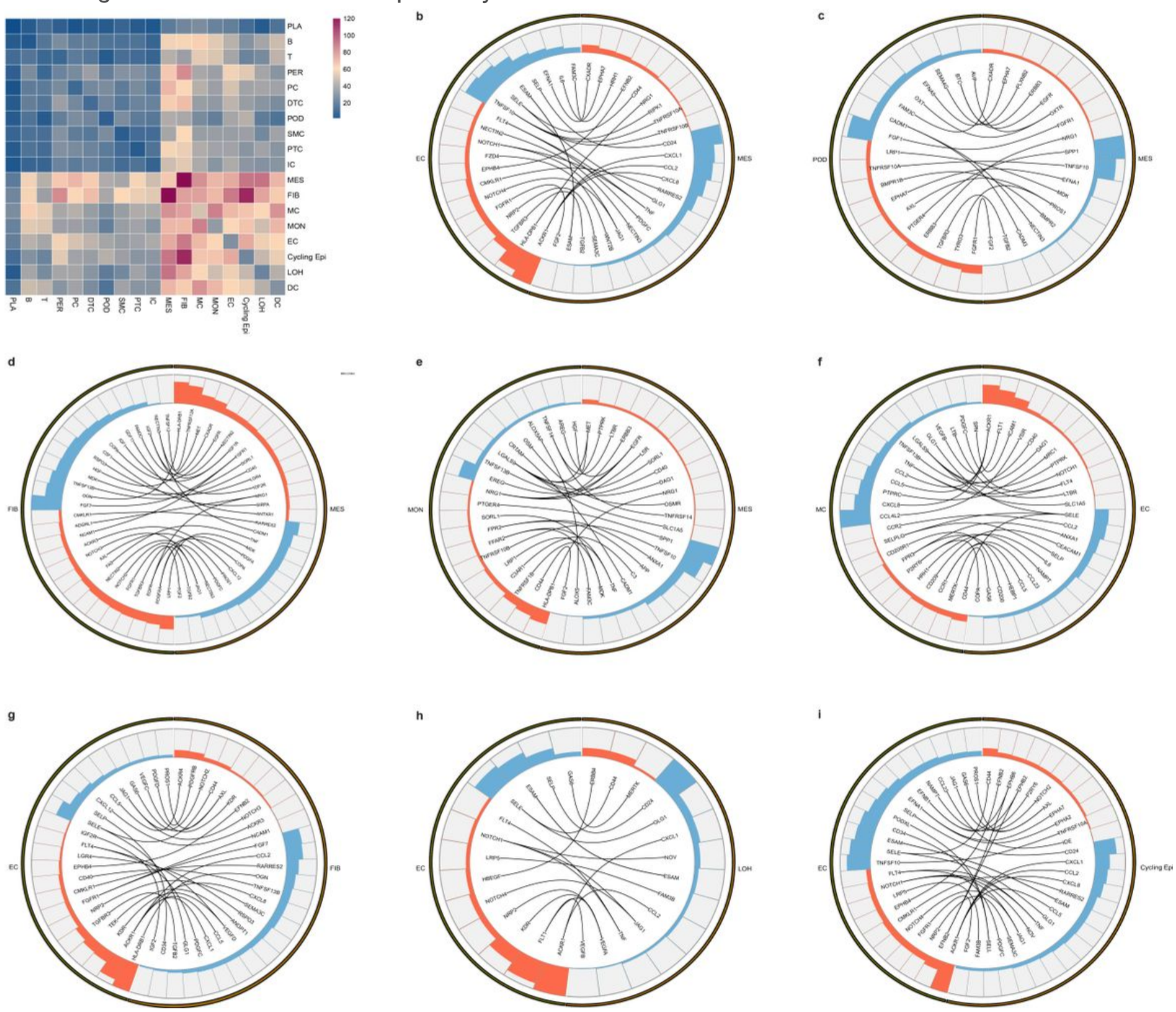

Figure 6

Cell-cell crosstalk between different cell clusters in the kidney of HTN patients. (a) Cell-cell communication events in kidney by analysis of ligand-receptor interactions between distinct cell types are shown. Visualized graphs indicate representative ligand-receptor interactions between mesangial cells and endothelial cells (b), podocytes (c), fibroblasts (d), or monocytes (e). Representative ligand-receptor interactions between endothelial cells and macrophages (f), fibroblasts $(\mathrm{g})$, loop of Henle cells $(\mathrm{h})$, or cycling epithelial cells (i). Lines represent interrelations between the ligand and corresponding receptor. Genes shown in blue represent ligands, and those depicted in red are receptors. Only HTN patients $(n=2)$ were analyzed. 


\section{Supplementary Files}

This is a list of supplementary files associated with this preprint. Click to download.

- SupplementalTable.1.doc

- Supplementaldataset.1.xls

- Supplementaldataset.2.xlsx

- Supplementaldataset.3.xlsx

- Supplementaldataset.4.xlsx

- Supplementaldataset.5.xlsx

- Supplementaldataset.6.xlsx

- Supplementaldataset.7.xls 